\title{
A Patient With Graves' Disease And Thyroid-Associ- ated Orbitopathy Undergoing Radioactive Iodine in Dr. Soetomo General Academic Hospital, Surabaya
}

\author{
Rizki Adrian Hakim ${ }^{1(\mathbb{D})}$, Stepanus Massora ${ }^{2}{ }^{(\mathbb{D})}$ Delfitri Lutfi $^{3}{ }^{(\mathbb{D}}$, Hermina Novida* $^{4 *}$ \\ ${ }^{1}$ Department of Internal Medicine, Faculty of Medicine, Universitas Airlangga - Dr. Soetomo General Academic Hospital, \\ Surabaya, Indonesia \\ ${ }^{2}$ Division of Nuclear Medicine, Department of Radiology, Faculty of Medicine, Universitas Airlangga - Dr. Soetomo \\ General Academic Hospital, Surabaya, Indonesia \\ ${ }^{3}$ Division of Orbital Ophthalmic Oncology, Department of Ophthalmology, Faculty of Medicine, Universitas Airlangga - \\ Dr. Soetomo General Academic Hospital, Surabaya, Indonesia \\ ${ }^{4}$ Division of Endocrinology, Department of Internal Medicine, Faculty of Medicine, Universitas Airlangga - Dr. Soetomo \\ General Academic Hospital, Surabaya, Indonesia
}

\section{A R T I C L E I N F O}

\section{Article history:}

Received 19 May 2021

Received in revised form 15 June 2021

Accepted 17 June 2021

Available online 30 June 2021

\section{Keywords:}

Hyperthyroidism,

Graves' disease,

Graves' ophthalmopathy,

Radioactive iodine, prophylaxis

*) Corresponding author:

hermina-n@fk.unair.ac.id

\begin{abstract}
A B S T R A C T
Graves' Disease (GD) is the most common etiology of thyrotoxicosis, followed by toxic multinodular goiter and toxic adenoma. GD can be managed with anti-thyroid drugs (ATDs), surgery, or radioactive iodine (RAI). Thyroid-associated orbitopathy (TAO) or Graves' Ophthalmopathy (GO) affects $25 \%-50 \%$ patients with GD, and its presence usually dissuade clinicians to use RAI in treating hyperthyroidism. The presence of GO is a relative contraindication use of RAI in patients with GD, as RAI can worsen existing GO. Corticosteroid prophylaxis can be given to such patients to reduce likelihood of worsening of GO. However, patient with moderate to severe active GO is currently advised against undergoing RAI. Established guidelines recommend the use of corticosteroid prophylaxis in these patients. We reported a patients with GD and orbitopathy who was treated with RAI and was given steroid prophylaxis to prevent worsening of GO.
\end{abstract}

\section{Introduction}

Graves' disease (GD) is the most common etiology of hyperthyroidism, which generally occurs in 4060 years' old patients. The underlying pathology of GD is the presence of TRab, an antibody against thyroid stimulating hormone (TSH), although the exact mechanism is still unknown. The antibody, an $\mathrm{IgG}$, binds to the thyrotropine receptors and activates it, thus leads to hyperthyroidism. This activation also stimulates follicular hypertrophy and hyerplasia, which causes gland enlargement and also increase of thyroid hormone synthesis. A study in the UK found the incidence of GD was $24 .{ }^{8}$ per 100000 people/year. The prevalence of GD was reported around $0.5 \%$ of the general population, with female predominance (5-10 : 1). ${ }^{1,2}$ In Indonesia, based on the Basic Health Research
2013, the prevalence of hyperthyroidism was 0.4 case per 10000 people. $^{3}$

Graves' ophthalmopathy (GO) is an orbital inflammation associated with GD, with incidence rate $25 \%-50 \%$ of all patients with GD. Symptoms of GO are corneal irritation, periocular edema, lid retraction, conjungtival erythema/chemosis, and extraocular muscles dysfunction. Some patients progress to more severe manifestation such as significant inflammation/ congestion, excessive proptosis, and corneal ulceration or optic neuropathy, which can lead to permanent blindess. ${ }^{4,5}$

According to guideline published by the American Thyroid Association (ATA) on management of GD, either antithyroid drugs (ATDs), thyrodectomy, or thyroid ablation using radioactive iodine (RAI) can 
be used as one of therapy modalities. The choice is based on patient's preference and clinical circumstance, since long-term quality of life between these therapies is not different. ${ }^{6}$ RAI is usually advised for patients who are unwilling to take lifelong medication or for those who cannot achieve euthyroidism with ATD. ${ }^{7}$ However, RAI is known to induce GO in GD patients with certain risk factors; therefore, GD patients with GO are usually given other choices of therapy. If RAI is given to GD patients with GO or those with risk factors, corticosteroid is indicated to prevent progression of GO after RAI. ${ }^{8}$

We present a case of a female patient with history of GD and GO, which was treated with RAI following her preference over ATD, which she had been consuming for years. She was then given corticosteroids to prevent progression of her $\mathrm{GO}$, and she responded well to medication.

Case

A 27-year-old female presented to the endocrinology outpatient with orbital pain since 1 month before presentation. She had chief a complaint of intermittent orbital pain since 5 years ago, but it was intensified since she treated with RAI 1 month ago. She also complained of painless lump on her right neck since 5 years ago. She denied having blurred vision nor double vision, but she felt protrusion of the right eye. She denied any fatigue, palpitations, diarrhea, excessive sweating, nor fever. She had been diagnosed with GD since 5 years ago, and had been taking thyrozol and propranolol. She also intermittenly takes methylprednisolone since 5 years ago whenever she felt any orbital pain. Her symptoms of thyrotoxicosis were well controlled with ATD which she had been taking for the last 4 years, but she sought another choice of therapy. Initially offered for thyroid surgery, however, she opted for RAI which was done 1 month prior to presentation. Her medication at presentation was thyrozol 5 milligrams once daily. Physical examination showed an alert condition, blood pressure $120 / 70 \mathrm{mmHg}$, pulse 86 beats per minute, respiratory rate 16 times per minute, peripheral oxygen saturation $98 \%$ on room air, axillary temperature $36.3^{\circ} \mathrm{C}$, and body weight of $55 \mathrm{~kg}$. Head and neck examination revealed a diffusely enlarged thyroid gland without signs of inflammation and a palpable, non-tender lump on right thyroid gland, measuring approximately $1 \mathrm{~cm}$ in diameter. From eye examinationboth eyes' visual acuity were $6 / 6$, there was lid retraction and lid lag on right eye, and no sign of conjunctival redness (Figure 1). Hertel exophthalmometer: right eye $20 \mathrm{~mm}$ and left eye $20 \mathrm{~mm}$. According to scoring for GO, she was in mild GO (NOSPECS scoring) and activity score 3 (CAS scoring). Other physical examinations were normal. Her thyroid function test was normal (FT4 $1.22 \mathrm{ng} / \mathrm{dL}$ dan TSH $0.743 \mathrm{ng} / \mathrm{dL}$ ), with TRAb $2.82 \mathrm{IU} / \mathrm{L}$ (1 year prior to presentation) and $6.01 \mathrm{IU} / \mathrm{L}$ (4 years prior to presentation) (Table 1). Ultrasound of the neck showed diffuse enlargement of thyroid gland, with a hypoechoic nodule measuring $2.3 \times 1.6 \mathrm{~cm}$ in the right lobe without calcification and vascularization. Fine needle aspiration was done on the nodule, which showed no malignant cells. Her thyroid scan showed a nodular goitre with non-toxic laboratory result (Figure 2). The head MSCT showed $0.5 \mathrm{~cm}$ proptosis of the right eye and hypertrophy of extraocular muscle, conforms with a thyroid-associated orbitopathy (Figure 3). She was diagnosed with GD (post RAI), mildly active GO, and benign thyroid nodule. She was given thyrozol 2.5 milligrams once daily and methylprednisolone 8 milligrams thrice daily, and she was told to follow-up the next month.

A month after her first visit, she came to the outpatient clinic with episodic palpitations, fatigue, and excessive swating. Her thyroid function tests were as follows: FT4 $1.23 \mathrm{ng} / \mathrm{mL}$, TSH $0.194 \mathrm{ng} / \mathrm{mL}$ (Table 1). She was given propranolol 10 milligrams once daily, and her other medications were continued.

The next month, she presented with much improved symptoms of thyrotoxicosis and orbital pain. Physical examination revealed right eye proptosis and lid retraction. Hertel exophthalmometer: right eye $16 \mathrm{~mm}$, left eye $20 \mathrm{~mm}$. Her CAS score was 1 . Her thyroid functions were as follows: FT4 $1.05 \mathrm{ng} / \mathrm{mL}$, TSH 4.815 $\mathrm{ng} / \mathrm{mL}$ (Table 1). Propranolol had been stopped then changed with thyrozol 2.5 milligrams every two days alongside with tapered down of methylprednisolon dose.

The following month, she came to the outpatient clinic with chest discomfort and fatigue without orbital pain which accordance with a clinical manifestation of hypothyroidism. Her vital signs were within normal limit. Physical examination revealed proptosis and lid retraction of the right eye. Hertel exophthalmometer: right eye $20 \mathrm{~mm}$, left eye $16 \mathrm{~mm}$, and her CAS score was 1. Her ATD had been stopped for 1 month. Her thyroid function tests were as follows: FT4 $1.03 \mathrm{ng} / \mathrm{mL}$ and TSH $11.197 \mathrm{ng} / \mathrm{mL}$ (Table 1). She was diagnosed with GD post RAI and subclinical hypothyroidism, then was given levothyroxine 50 microgram once daily. Her subsequent visits were symptom-free and her thyroid functions were within normal range under the daily levothyroxine, and she was scheduled for eyelid surgery to correct the lid retraction. Table 1 summarizes her thyroid function throughout monthly visits.

Table 1. Patient's thyroid function throughout her monthly visits

\begin{tabular}{lcccccccc}
\hline & $\begin{array}{c}29 / 11 / 14 \\
\text { (4 years ago) }\end{array}$ & $\begin{array}{c}12 / 9 / 17 \\
\text { (after ATD) }\end{array}$ & $\begin{array}{c}12 / 1 / 18 \\
\text { (before RAI) }\end{array}$ & $\begin{array}{c}10 / 2 / 18 \\
\text { (1st visit) }\end{array}$ & $\begin{array}{c}\text { (2nd visit) } \\
\text { (3rd visit) }\end{array}$ & $\begin{array}{c}30 / 6 / 18 \\
\text { (4th visit) }\end{array}$ & $\begin{array}{c}27 / 7 / 18 \\
\text { (5th visit) }\end{array}$ \\
\hline FT4 (ng/dL) & 3.05 & & 1.12 & 1.22 & 1.23 & 1.05 & 1.03 & 0.82 \\
\hline TSH (ng/dL) & $<0.01$ & & 1.265 & 0.743 & 0.194 & 4.815 & 11.197 & 3.3 \\
\hline TRAb (U/l) & 6.01 & 2.82 & & & & & \\
\hline
\end{tabular}




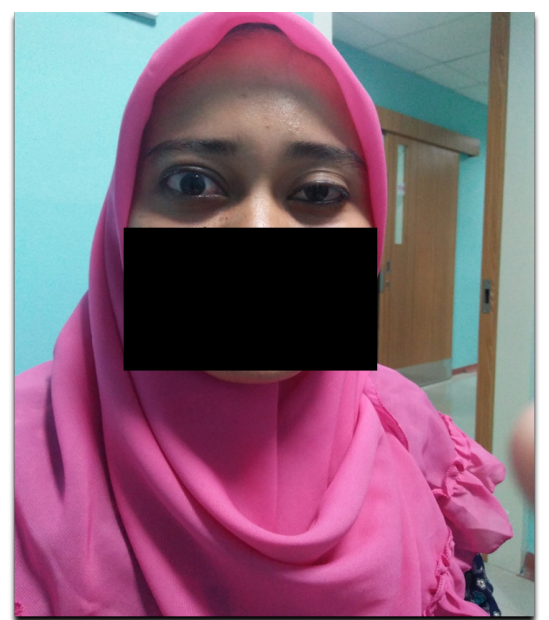

Figure 1. Clinical appearance of the patient during her first visit to endocrinology outpatient. Note the ptosis of left upper eyelid.

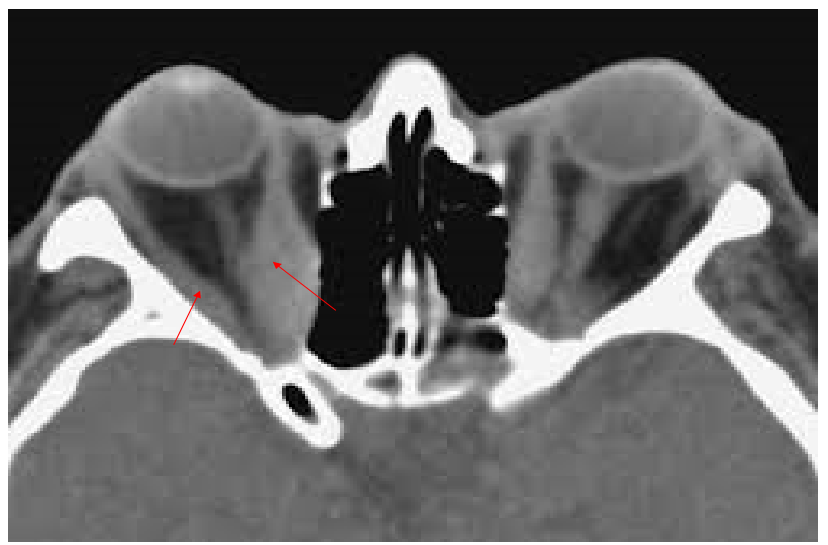

Figure 2. Patient's Head CT (Orbital-Focused). There is marked hypertrophy of medial and lateral right rectus muscles (red arrows), which pushes ocular bulb anteriorly, which causes proptosis $+/-0,5 \mathrm{~cm}$. Radiologist's remark: Thyroid-Associated Orbitopathy

\section{Discussion}

Graves' Disease is an autoimmune disease mediated by autoantibody, with thyrotoxicosis as its cardinal manifestation. GD is diagnosed by the presence of diffuse enlargement of the thyroid gland, moderate to severe hyperthyroidism, and orbitopathy. When these signs and symptoms are present, the diagnosis of GD is usually straightforward. In the absence of these features, elevated $\mathrm{TRAb}$ and characteristic thyroid scan (RAIU) uptake can aid in the diagnosis. ${ }^{9}$ Although GD is commonly known to involve thyroid gland, the other organ systems can be affected as well, either as a part of the autoimmune process or as complications of thyrotoxicosis. Systemic autoimmune involvement of GD includes eye (GO) and skin (Graves' dermatopathy), as tissues in these organs possess receptor to TRAb. ${ }^{10}$

Graves' ophthalmopathy (GO) is define as an inflammation of orbita associated with GD, however, not all GD patients will develop GO. GD patients with established GO or suspicion of GO must be evaluated by ophthalmologist. In terms of severity, GO can be classified into mild, moderate, and severe, according to NOSPECS (No physical signs or symptoms, Only signs, no symptoms, Soft tissue involvement, Proptosis, Extraocular muscle invlovement, Corneal involvement, Sight loss) criteria or EUGOGO (EUropean Groups On Graves' Orbitopathy) criteria. In terms of disease activity, GO can be divided into active or inactive based on CAS (Clinical Activity Score) scoring system. This classification will guide clinicians to determine the best course of action in treating the ophthalmopathy, with overall goal is to avoid visual-theratening disease and to achieve minimal disease activity. ${ }^{5}$

In GD patients with GO, therapy of GD with RAI should be done with consideration, because RAI can worsen the existing ophthalmopathy, especially when the patient develops symptomatic hypothyroidism after RAI. Guideline from ATA recommends systemic corticoteroids be given to GD patients with GO under RAI treatment within the setting of mild and active ophthalmopathy, with/without risk factors of worsening of GO. RAI is not recommended for GD patiens with moderate to severe $\mathrm{GO}{ }^{8}$

A RCT recommends giving 0.4-0.5 mg/kgBW/ day of prednisone as prophylaxis for GD patients with ophthalmopathy treated wih RAI, which can be started as early as 1-3 days after RAI. The dose should be given for 1 month, followed by 2 months of gradual tapering. In the other hand, a retrospective cohort study reported that lower dose and shorter duration of steroid (prednison $0.2 \mathrm{mg} / \mathrm{kgBW} /$ day for 6 weeks) has a similar efficacy in terms of preventing new onset GO and worsening of ophthalmopathy in patients with preexisting GO. Currently, most guidelines recommend administering 30 $\mathrm{mg}$ of prednisone daily followed by tapering down, with total duration of 6-8 weeks. After achieving a low disease activity, any residual anatomical disruption of the orbita can be corrected by surgery. ${ }^{8}$

\section{Conclusion}

In summary, RAI can be chosen as therapy for patients with GD presenting with mild and active GO, as per 2016 guideline from American Thyroid Association. Prophylactic steroid is given after RAI to prevent worsening of GO. PostRAI care should be given to prevent hypothyroidism after RAI, which can also worsen GO. This patient was treated with RAI over surgery after taking ATD for years despite her ophthalmopathy, and was given prophylactic steroid. She achieved hypothyroidism several months after RAI, and did not show any deterioration of ophthalmopathy.

\section{Conflict of Interest}

The author stated there is no conflict of interest

\section{References}

1. Hussain YS, Hookham JC, Allahabadia A, Balasubramanian SP. Epidemiology, management and outcomes of Graves' disease-real life data. Endocrine. 2017;56(3):568-578. doi:10.1007/s12020-017-13065

2. Brent GA. Graves' Disease. N Engl J Med. 2008;358(24):2594-2605. doi:10.1056/NEJMcp0801880

3. Badan Penelitian dan Pengembangan Kesehatan. Indonesian Basic Health Research 2013. Lap Nas 2013. 2013:1-384. doi:1 Desember 2013

4. Bahn RS. Graves' ophthalmopathy. N Engl J Med. 2010;362(8):726738. doi:10.1056/NEJMra0905750

5. Tanda ML, Piantanida E, Liparulo L, et al. Prevalence and natural history of graves' orbitopathy in a large series of patients with newly diagnosed graves' hyperthyroidism seen at a single center. J Clin Endocrinol Metab. 2013;98(4):1443-1449. doi:10.1210/jc.2012-3873 
6. Abraham-Nordling M, Törring O, Hamberger B, et al. Graves' disease: a long-term quality-of-life follow up of patients randomized to treatment with antithyroid drugs, radioiodine, or surgery. Thyroid. 2005;15(11):1279-1286. doi:10.1089/thy.2005.15.1279

7. Walter MA, Briel M, Christ-Crain M, et al. Effects of antithyroid drugs on radioiodine treatment: Systematic review and meta-analysis of randomised controlled trials. Br Med J. 2007;334(7592):514-517. doi:10.1136/bmj.39114.670150.BE

8. Ross DS, Burch HB, Cooper DS, et al. 2016 American Thyroid Association Guidelines for Diagnosis and Management of Hyperthyroidism and Other Causes of Thyrotoxicosis. Thyroid. 2016;26(10):1343-1421. doi:10.1089/thy.2016.0229

9. Bahn RS, Burch HB, Cooper DS, et al. Hyperthyroidism and Other Causes of Thyrotoxicosis: Management Guidelines of the American Thyroid Association and American Association of Clinical Endocrinologists. Thyroid. 2011;21(6):593-646. doi:10.1089/ thy.2010.0417

10. Barbesino G, Tomer Y. Clinical review: Clinical utility of TSH receptor antibodies. J Clin Endocrinol Metab. 2013. doi:10.1210/jc.2012-4309 